\section{Kompass \\ Autoimmun}

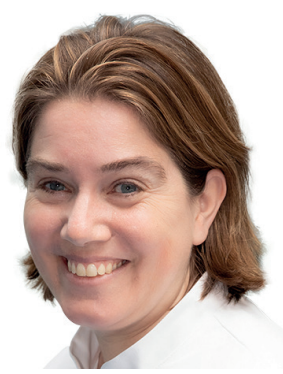

\section{Caroline Bosch-Voskens}

Hautklinik, Universitätsklinikum Erlangen/ Friedrich-Alexander-Universität ErlangenNürnberg, Erlangen, Deutschland

\title{
Sechs Fragen an
}

\section{PD Dr. Caroline Bosch-Voskens,}

\section{Preisträgerin des Oscar-Gans-Förderpreises 2021}

\section{Akademischer Werdegang}

- Seit 2020 Leiterin der Phase-I-Studie «Sicherheit und Verträglichkeit von autologen ex vivo expandierten regulatorischen T-Zellen bei Erwachsenen mit Colitis ulcerosa»

- Seit 2018 Arbeitsgruppenleiterin im SFB/TransRegio 241 «Immun-epitheliale Signalwege bei chronisch-entzündlichen Darmerkrankungen»

- Habilitation in der Hautklinik des Universitätsklinikums Erlangen

- Ausbildung zur Fachärztin für Haut- und Geschlechtskrankheiten an der Hautklinik des Universitätsklinikums Erlangen

- Promotion an der Universität Groningen, Niederlande

- Research Fellowship an der University of Maryland School of Medicine, Baltimore, USA

- Studium der Humanmedizin an der Universität Leiden, Niederlande

\section{Forschungsschwerpunkte}

- Beweglichkeit und Migration von Immunzellen in 3-D

- Zellbasierte Therapie mit regulatorischen T-Zellen bei Patienten mit chronisch-entzündlichen Erkrankungen

- Ex vivo expandierte NK-Zellen für die Behandlung von Melanom

Sie wurden für Ihre Erforschung der Beeinflussung der Beweglichkeit natürlicher Killerzellen (NK) durch Kryopräservation, einer kontrollierten Tiefkühlung, geehrt. Welche Resultate haben Sie dabei am meisten überrascht?

In der prämierten Arbeit haben wir eindeutig zeigen können, dass die breit etablierten und genutzten Standard-Zytotoxizitätsassays nicht wie bisher angenommen die reale Funktionalität wiedergeben, sondern dass relevante Unterschiede auf zellulärer Ebene durch die Vorgehensweise in den bisher genutzten Standardassays maskiert werden. Die Interpretation dieser «Standard»-Assays ist daher sorgfältig abzuwägen und ggf. sind ergänzende Assays notwendig.

Worin besteht die therapeutische Bedeutung Ihrer Forschung kurz- oder auch mittelfristig? Da die Kryokonservierung die Beweglichkeit der NK-Zellen negativ beeinflusst, ist mit einer verminderten Migration der Zellen im menschlichen Körper nach adoptivem Zelltransfer zu rechnen. Wir gehen davon aus, dass hierdurch die klinische Nutzung von zellbasierten 
Produkten zur Behandlung von Erkrankungen, bei denen eine Migration im Gewebe (zum Beispiel bei entzündlichen Hauterkrankungen oder soliden Tumoren) essentiell ist, generell beeinträchtigt wird.

Die von Ihnen verwendeten Zellen stammten aus einem klinischen Setting. Waren Zellen von chronisch-entzündlichen Erkrankungen oder Autoimmun-Erkrankungen beteiligt? Was lässt sich für diese Erkrankungen aus Ihrer Forschung schlussfolgern?

In der prämierten Arbeit wurden lediglich Zellen von gesunden Probanden verglichen. Aktuell laufen jedoch Versuche, bei denen ex vivo expandierte regulatorische T-Zellen (Treg) von Patienten mit chronisch-entzündlichen oder Autoimmun-Erkrankungen hinsichtlich Beweglichkeit und Migration in 3-D untersucht werden. Diese Versuche sind für diese Patienten relevant, da Treg die Immunität hemmen und durch den adoptiven Treg-Transfer Entzündungen gehemmt werden können und das immunologische Gleichgewicht hierdurch wiederhergestellt werden kann.

Sie selbst sind Fachärztin für für Haut- und Geschlechtskrankheiten. Mit welchen Fachrichtungen arbeiten Sie bei Ihrer Arbeit interdisziplinär zusammen?

Ich arbeite in der klinischen Patientenversorgung sehr eng mit allen internistischen Kliniken und den chirurgischen Abteilungen zusammen. Über das interdisziplinäre Tumorboard besteht eine sehr enge Kooperation mit der Radiologie, Chirurgie, Strahlentherapie, Nuklearmedizin und internistischen Onkologie. In der Forschung bin ich mit einem klinisch-translationalen Projekt in der SFB/TransRegio 241 «Immun-epitheliale Signalwege bei chronisch-entzündlichen Darmerkrankungen» zusammen mit Prof. Markus Neurath (Medizinische Klinik 1, Universitätsklinikum Erlangen) beteiligt. In diesem Projekt werden weltweit erstmalig Patienten mit Colitis ulcerosa mit körpereigenen ex vivo expandierten regulatorischen Treg behandelt und die Sicherheit und Verträglichkeit dieses sog. adoptiven Treg-Transfers untersucht. Darüber hinaus besteht eine sehr produktive Zusammenarbeit mit der Arbeitsgruppe von Prof. Ben Fabry (Lehrstuhl für Biophysik), wie wir in der prämierten Forschungsarbeit zeigen konnten.

\section{Was bedeutet diese Auszeichnung für Sie?}

Die Auszeichnung ist für mich die Belohnung für den jahrelangen Spagat zwischen Klinik und Forschung und motiviert mich sehr, diesen dualen Karriereweg weiter zu verfolgen. Des Weiteren sehe ich die Auszeichnung auch als Anerkennung unserer aktuellen Forschungsideen, welche innovativ und sicherlich etwas «outside the box» sind.

\section{Womit finden Sie einen Ausgleich für Ihre Forschungstätigkeit in der Klinik?}

Zwischen den Schafen auf unserer kleinen Streuobstwiese schalte ich am Wochenende vom Alltag ab. Die physische Arbeit in der Natur und die Stille der Umgebung sind für mich die ideale Voraussetzung, um meine Gedanken zu ordnen und neue Ideen und Hypothesen zu überdenken. Wenn ich anschließend mit meiner kleinen Familie das Lagerfeuer anzünde und wir gemeinsam den Tag ausklingen lassen, bin ich sehr glücklich und entspannt.

Frau Dr. Bosch-Voskens, herzlichen Dank für das Interview!

\section{ZUM PREIS}

Der Oscar-Gans-Preis ist eine der bedeutendsten Auszeichnungen der Dermatologie und wird alle zwei Jahre von der Deutschen Dermatologischen Gesellschaft (DDG) ausgeschrieben. Der Hauptpreis fördert die experimentelle Dermatologie und ist mit 15000 Euro dotiert. Zudem werden jedes Jahr Förderpreise vergeben, die mit je 5000 Euro dotiert sind. Teilnehmen können Hautärztinnen und -ärzte sowie Forscherinnen und Forscher im Bereich der Dermatologie. 